\title{
Compatibilidade de inseticidas utilizados no controle da mosca branca em soja com Beauveria bassiana
}

\author{
Roberta Pacola Oliveira ${ }^{1}$, Luis Gustavo Amorim Pessoa ${ }^{1}$, Elisângela de Souza Loureiro ${ }^{1}$, \\ Marcela Pacola Oliveira ${ }^{1}$ \\ ${ }^{1}$ Universidade Federal de Mato Grosso do Sul, Campus de Chapadão do Sul, Chapadão do Sul, Mato Grosso do Sul, Brasil. E-mail: \\ robertapacola@hotmail.com, luis.pessoa@ufms.br, elisangela.loureiro@ufms.br,marcela_pacola@hotmail.com
}

Recebido: 08/02/2018; Aceito: 16/10/2018.

\begin{abstract}
RESUMO
Objetivou-se avaliar o efeito de inseticidas utilizados no manejo da mosca branca sobre o fungo entomopatogênico Beauveria bassiana. Os bioensaios foram realizados in vitro, utilizando inseticidas em doses mínima, máxima e média, quando possível. Avaliou-se o crescimento vegetativo, a produção de conídios e a compatibilidade dos produtos com o fungo. Apenas o inseticida contendo piriproxifem na dose máxima não interferiu negativamente na produção de conídios. Todos os inseticidas e doses testadas reduziram significativamente a germinação dos conídios. Somente o inseticida contendo espiromesifeno, na dose média, proporcionou crescimento vegetativo significativamente superior à testemunha, possibilitando sua classificação como compatível com o fungo B. bassiana.
\end{abstract}

Palavras-chave: Bemisia tabaci, controle microbiano, fungo entomopatogênico, produto fitossanitário, Glicyne $\max$.

\section{Compatibility of insecticides used for the whitefly control on soybean with Beauveria bassiana}

\begin{abstract}
The objective of this study was to evaluate the effect of insecticides used in the management of the whitefly on the entomopathogenic fungus Beauveria bassiana. The bioassays were performed in vitro, using insecticides at minimum, maximum and average doses, when possible. Vegetative growth, conidia production and the compatibility of the products with the fungus were evaluated. Only the insecticide containing pyriproxine at maximum dose did not adversely interfere with the production of conidia. All insecticides and doses tested significantly reduced conidial germination. Only the insecticide containing spiromesifen product in the medium dose provided a vegetative growth significantly superior to the control, allowing its classification as compatible with the B. bassiana fungus.
\end{abstract}

Keywords: Bemisia tabaci, microbial control, entomopathogenic fungus, phytosanitary product, Glicyne max. 
A mosca branca Bemisia tabaci Gennadius (Hemiptera: Aleyrodidae) foi, por muito tempo, considerada praga ocasional em soja no Brasil. Todavia, o biótipo B de B. tabaci, introduzido no início da década de 90 , vem se tornando mais importante a cada safra. Em função deste fato, os agricultores têm utilizado medidas de controle químico para seu manejo (Lima e Lara, 2004), com consequências que podem ser desastrosas para o Manejo Integrado de Pragas (MIP). O principal método de controle desse inseto é a aplicação de inseticidas químicos, que pode proporcionar a seleção de populações resistentes desta praga. Desta forma, há necessidade de estudos visando o desenvolvimento de novas estratégias e alternativas para seu manejo (Baldin et al., 2005).

Uma dessas alternativas é a utilização de produtos fitossanitários seletivos/compatíveis, que promovam a preservação dos inimigos naturais e, ao mesmo tempo, controlem os insetos praga (Newsom et al., 1976). A seletividade/compatibilidade nos programas de MIP é um conceito importante no momento da escolha do inseticida ou da maneira de aplicá-lo, visando a preservação dos inimigos naturais, espécies inofensivas à cultura e a outros organismos benéficos que convivem no agroecossistema. A aplicação de produtos fitossanitários não deve afetar apenas a população de entomófagos (Yamamoto e Bassanezi, 2003), mas também os entomopatógenos, visto que várias espécies destes atuam no controle da população de insetos que causam danos econômicos às culturas, visando minimizar os prejuízos por eles causados. Dentre esses microrganismos, destacam-se os fungos entomopatogênicos como alternativa viável no controle de insetos no país (Onofre et al., 2001; Silva et al., 2009).

Os fungos são organismos de tamanho e formas variáveis, que podem ser unicelulares como as leveduras ou constituídos por um conjunto filamentoso de micélio. Beauveria bassiana Vuillemin é uma espécie de fungo entomopatogênico, cujo ciclo biológico permite sua caracterização como um parasita facultativo. Seus conídios podem penetrar em qualquer parte da cutícula do inseto (Alves, 1998). Este microrganismo é empregado em escala comercial em alguns países, dentre eles Estados Unidos e México (Faria, 2001). No Brasil, já existem produtos comerciais à base desse entomopatógeno, recomendado para o manejo de $B$. tabaci (MAPA/Agrofit, 2017).

A utilização associada de produtos fitossanitários e fungos entomopatogênicos para o controle de pragas nas lavouras é capaz de auxiliar na redução da sua população e incidência, devido à presença de substâncias contidas nos produtos fitossanitários que conseguem atuar como estressantes, favorecendo então a infecção de fungos que realizam o controle biológico de pragas (Sosa-Gomez, 2005).

Diante do exposto, este trabalho teve como objetivo avaliar a compatibilidade in vitro entre inseticidas com diferentes ingredientes ativos recomendados para o controle da mosca branca na cultura da soja com o fungo entomopatogênico B. bassiana.

Para o experimento, utilizou-se o produto comercial contendo o fungo $B$. bassiana (Isolado IBCB 66). Os testes foram realizados in vitro avaliando-se efeitos sobre a germinação, o crescimento vegetativo e a produção de conídios na presença dos diferentes inseticidas e doses. Foram utilizados inseticidas recomendados para o controle da mosca branca na cultura da soja (Agrofit, 2017), fixando-se as doses mínima, máxima e média, quando possível, de acordo com as especificações dos fabricantes, com volume de calda fixo de $200 \mathrm{~L} \mathrm{ha}^{-1}$. O tratamento testemunha consistiu apenas em água destilada esterilizada (ADE) (Tabela 1).

Tabela 1 - Inseticidas recomendados para controle da mosca branca na cultura da soja.

\begin{tabular}{|c|c|c|c|}
\hline Ingrediente Ativo & Grupo Químico & Formulação & $\begin{array}{c}\text { Doses } \\
\left(\mathrm{L} \mathrm{ha}^{-1}\right)\end{array}$ \\
\hline Testemunha & - & - & - \\
\hline \multirow{3}{*}{ Piriproxifem (T) } & \multirow{3}{*}{ Éter Piridiloxipropílico } & \multirow{3}{*}{$\mathrm{CE} *$} & 0,250 \\
\hline & & & 0,275 \\
\hline & & & 0,300 \\
\hline Piriproxifem $(\mathrm{C})$ & Éter Piridiloxipropílico & $\mathrm{CE} *$ & 0,250 \\
\hline \multirow{3}{*}{ Espiromesifeno } & \multirow{3}{*}{ Cetoenol } & \multirow{3}{*}{$\mathrm{SC} * *$} & 0,400 \\
\hline & & & 0,500 \\
\hline & & & 0,600 \\
\hline \multirow{3}{*}{ Beta- ciflutrina + Imidacloprido } & \multirow{3}{*}{ Piretroide + Neonicotinoide } & \multirow{3}{*}{$\mathrm{CE} *$} & 0,250 \\
\hline & & & 0,275 \\
\hline & & & 0,300 \\
\hline Thiamethoxam + Lambdacialotrina & Neocotinoide + Piretroide & $\mathrm{SC} * *$ & 0,250 \\
\hline Imidacloprido & Neonicotinoide & $\mathrm{SC} * *$ & 0,250 \\
\hline
\end{tabular}


Para que fossem efetuadas as avaliações do crescimento vegetativo e a produção de conídios do fungo, acrescentou-se, proporcionalmente, $200 \mathrm{~mL}$ do meio nutritivo (BDA: batata-dextrose-ágar) ainda não solidificado dos inseticidas, com suas respectivas dosagens a $40^{\circ} \mathrm{C}$. Em seguida, a mistura foi vertida em placas de Petri com nove centímetros de diâmetro, devidamente identificadas de acordo com os tratamentos propostos. Após a solidificação do meio, procedeu-se à inoculação do fungo por meio de alça de platina em três pontos equidistantes. Em seguida, as placas foram lacradas com filme PVC e acondicionadas em câmara climatizada tipo BOD à temperatura de $25 \pm 1{ }^{\circ} \mathrm{C}$, umidade relativa de $70 \pm 10 \%$ e fotofase de 12 horas, permanecendo nessas condições por sete dias para promover o desenvolvimento vegetativo e reprodutivo do fungo (Alves et al., 1998).

Para avaliação do crescimento vegetativo, foram realizadas medições das colônias em sentidos ortogonais, obtendo-se, posteriormente, o diâmetro médio em centímetros. Para análises da produção de conídios, as colônias foram recortadas das placas com auxílio de um bisturi cirúrgico estéril e transferidas para tubos de ensaio esterilizados, contendo $10 \mathrm{~mL}$ de água destilada esterilizada e espalhante adesivo (Tween ${ }^{\circledR} 80$ ). Em seguida, foi realizada a agitação manual e vigorosa para que houvesse a separação dos agregados. Logo após, realizou-se diluições sucessivas (quando necessário) e a contagem de conídios em microscópio óptico com o auxílio de uma câmara Neubauer. O delineamento estatístico utilizado foi inteiramente casualizado com 13 tratamentos (testemunha e inseticidas nas diferentes doses), cada um composto por três placas, totalizando nove colônias. Dessas, apenas seis foram aleatoriamente apontadas, resultando assim em seis repetições por tratamento (Alves, 1998).

Para avaliação da germinação, utilizou-se suspensão de $1,0 \times 10^{8}$ conídios $\mathrm{mL}^{-1}$, a qual foi mantida em repouso por duas horas em caldas contendo os diferentes inseticidas e doses. Após esse tempo, foi plaqueada $1,0 \mathrm{~mL}$ da suspensão com auxílio de pipeta graduada ( $1 \mathrm{~mL}$ ) em placas de Petri (5 por tratamento) e espalhada com alça Drigalsky no meio nutritivo (BDA). Após esse processo, as placas foram identificadas, lacradas com filme PVC e incubadas por 20 horas em câmara climatizada tipo BOD a $25 \pm 1^{\circ} \mathrm{C}$, umidade relativa de $70 \pm 10 \%$ e fotofase de 12 horas. Após a incubação, as placas foram divididas em dois quadrantes, sendo contados, aleatoriamente, 100 conídios em cada um deles, estabelecendo, posteriormente, a porcentagem de germinação (Alves et al., 1998).

Em todos os bioensaios o delineamento foi inteiramente casualizado. Os dados de crescimento vegetativo e reprodutivo foram transformados para $(x+0,5)^{1 / 2}$ e, os dados de germinação, para arcsen $(\mathrm{x} / 100)^{1 / 2}$, sendo submetidos ao teste de agrupamento de médias de Scott-Knott a 5\% de probabilidade. Os dados obtidos também foram utilizados para se calcular o fator de compatibilidade (IB = índice biológico) proposto por Rossi-Zalaf et al. (2008), que permite a classificação dos produtos de acordo com o efeito observado em relação aos parâmetros avaliados.

Em relação ao crescimento vegetativo de $B$. bassiana, verificou-se que os tratamentos betacyfluthrin + imidacloprido na dose média e espiromesifeno na dose mínima foram semelhantes à testemunha. $\mathrm{O}$ tratamento espiromesifeno na dose média proporcionou crescimento vegetativo significativamente superior e os demais, inferiores à testemunha, sendo piriproxifem (C) e piriproxifem (T), em todas as dosagens, aqueles que proporcionaram reduções mais significativas nos valores desse parâmetro (Tabela 2).

Avaliando-se a produção de conídios, verificou-se que apenas piriproxifem (T) na dose máxima foi semelhante à testemunha. Os demais foram significativamente inferiores, sendo os tratamentos piriproxifem (C), piriproxifem (T) na dose mínima, beta-cyfluthrin + imidacloprido e espiromesifeno (em todas as dosagens) os que proporcionaram reduções mais significativas nos valores desse parâmetro (Tabela 2).

Em trabalho realizado por Almeida et al. (2003) foi avaliada a compatibilidade de produtos fitossanitários químicos na conservação de microrganismos entomopatogênicos no manejo de pragas do cafeeiro, no qual foi verificado que o fungo B. bassiana isolado 634 teve o seu crescimento vegetativo afetado pelas duas concentrações de cyproconazole + thiamethoxam (200 $\mathrm{g} \mathrm{ha}^{-1}$ ) $20 \mathrm{GR}$ e endosulfan CE, sendo estes significativamente inferiores aos demais tratamentos e à testemunha.

A esporulação foi afetada por todos os produtos testados, porém, as doses de cyproconazole + thiamethoxam 20 GR e endosulfan CE utilizadas proporcionaram efeito mais significativo, chegando a impedir a esporulação do fungo, semelhante ao resultado obtido no presente trabalho em relação ao produto combinado que contém o ingrediente ativo thiamethoxam.

Ao se avaliar a germinação, verificou-se que todos os inseticidas e dosagens testadas interferiram significativamente nos valores desse parâmetro, pois foram consideravelmente inferiores à testemunha. Em relação, especificamente, aos produtos que contêm piriproxifem, verifica-se, pelos resultados obtidos, maior efeito inibitório deste ingrediente ativo e possível efeito da formulação, sendo verificada diferença significativa entre as marcas comerciais testadas (Tabela 2). 
Tabela 2. Crescimento vegetativo ( $\mathrm{cm} \pm \mathrm{EP})$, produção de conídios $\left(\times 10^{8} \pm \mathrm{EP}\right)$ e germinação $(\% \pm \mathrm{EP})$ de B. bassiana na presença de diferentes inseticidas. Chapadão do Sul-MS, Brasil.

\begin{tabular}{|c|c|c|c|c|}
\hline Tratamentos & $\begin{array}{l}\text { Doses } \\
\left(\mathrm{L} \mathrm{ha}^{-1}\right)\end{array}$ & $\begin{array}{l}\text { Crescimento } \\
\text { Vegetativo }^{1}\end{array}$ & $\begin{array}{l}\text { Produção de } \\
\text { Conídios }^{1}\end{array}$ & Germinação $^{2}$ \\
\hline Testemunha & - & $1,75 \pm 0,12 b$ & $0,51 \pm 0,10 \mathrm{a}$ & $95,75 \pm 2,15 \mathrm{a}$ \\
\hline Piriproxifem (T) & 0,250 & $0,80 \pm 0,09 \mathrm{e}$ & $0,34 \pm 0,08 \mathrm{~b}$ & $20,50 \pm 1,18 \mathrm{f}$ \\
\hline Piriproxifem (T) & 0,275 & $0,74 \pm 0,10 \mathrm{e}$ & $0,06 \pm 0,01 \mathrm{c}$ & $20,87 \pm 1,09 \mathrm{f}$ \\
\hline Piriproxifem $(\mathrm{T})$ & 0,300 & $0,74 \pm 0,09 \mathrm{e}$ & $0,48 \pm 0,12 \mathrm{a}$ & $28,00 \pm 2,27 \mathrm{e}$ \\
\hline Piriproxifem (C) & 0,250 & $0,77 \pm 0,10 \mathrm{e}$ & $0,19 \pm 0,04 \mathrm{c}$ & $67,62 \pm 1.88 \mathrm{~d}$ \\
\hline Espiromesifeno & 0,400 & $1,51 \pm 0,08 \mathrm{c}$ & $0,17 \pm 0,04 \mathrm{c}$ & $80,37 \pm 1,97 \mathrm{c}$ \\
\hline Espiromesifeno & 0,500 & $2,21 \pm 0,10 \mathrm{a}$ & $0,11 \pm 0,03 \mathrm{c}$ & $80,87 \pm 2,02 \mathrm{c}$ \\
\hline Espiromesifeno & 0,600 & $1,68 \pm 0,13 b$ & $0,06 \pm 0,01 \mathrm{c}$ & $77,00 \pm 1,98 \mathrm{c}$ \\
\hline Beta-ciflutrina + Imidacloprido & 0,250 & $1,73 \pm 0,07 \mathrm{~b}$ & $0,09 \pm 0,01 \mathrm{c}$ & $78,87 \pm 1,97 \mathrm{c}$ \\
\hline Beta-ciflutrina + Imidacloprido & 0,275 & $1,69 \pm 0,09 \mathrm{~b}$ & $0,10 \pm 0,02 \mathrm{c}$ & $78,37 \pm 1,87 \mathrm{c}$ \\
\hline Beta-ciflutrina + Imidacloprido & 0,300 & $1,33 \pm 0,14 \mathrm{c}$ & $0,24 \pm 0,05 c$ & $86,25 \pm 2,12 b$ \\
\hline Thiamethoxam + Lambdacialotrina & 0,250 & $1,09 \pm 0,08 \mathrm{~d}$ & $0,29 \pm 0,06 \mathrm{~b}$ & $76,72 \pm 2,03 \mathrm{c}$ \\
\hline Imidacloprido & 0,250 & $1,20 \pm 0,12 \mathrm{~d}$ & $0,23 \pm 0,03 \mathrm{c}$ & $86,87 \pm 2,01 b$ \\
\hline $\mathrm{CV}(\%)$ & & 5,92 & 8,91 & 6,10 \\
\hline
\end{tabular}

Médias seguidas pela mesma letra minúscula na coluna não diferem significativamente entre si.

${ }^{1}$ Para análise dados transformados em $(x+0,5)^{1 / 2}$.

${ }^{2}$ Para análise dados transformados em $(\mathrm{x} / 100)^{1 / 2}$.

Ao realizar o cálculo do Índice Biológico verificouse que os inseticidas contendo imidaclopride, thiamethoxam + lambdacialotrina e piriproxifem $(\mathrm{T})$ (nas diferentes doses) foram considerados tóxicos. Betacyfluthrin + imidacloprido (nas diferentes doses) e espiromesifeno (nas doses mínima e máxima) foram medianamente tóxicos. $\mathrm{O}$ único tratamento compatível com o fungo $B$. bassiana foi o espiromesifeno na dose média (Tabela 3).

Em trabalho realizado por Moino Junior e Alves (1998), avaliando-se o efeito fungitóxico de Imidaclopride sobre a B. bassiana (isolado 634), foi observado menor efeito no diâmetro de colônias, proporcionando valores semelhantes à testemunha. Quando esses autores avaliaram o número de conídios produzidos, verificaram valor significativamente inferior à testemunha. Os resultados observados na presente pesquisa divergem dos por eles apresentados no que se refere ao crescimento vegetativo com produto contendo o mesmo ingrediente ativo, verificando-se redução significativa no valor desse parâmetro em relação à testemunha; já para a produção de conídios houve concordância dos resultados. Segundo Roberts e Campbell (1977), a suscetibilidade de fungos entomopatogênicos a produtos fitossanitários sintéticos apresenta variabilidade entre os diferentes agroquímicos e isolados de um mesmo fungo, explicando a variação nos resultados obtidos por diferentes autores. De acordo com Anderson e Roberts (1983), as formulações de inseticidas do tipo concentrado emulsionável (CE) estão, frequentemente, associadas com a inibição da germinação dos conídios de $B$. bassiana, ao contrário das formulações como pós molháveis (PM), que, geralmente, não promovem inibição e favorecem, muitas vezes, o crescimento deste patógeno.

Loureiro et al. (2002) estudaram o efeito de produtos fitossanitários sintéticos utilizados em alface e crisântemo sobre os fungos entomopatogênicos $B$. bassiana (isolado CB 66), Metarhizium anisopliae (Metsch) Sorok (isolado CB 121) e Isaria fumosorosea Wize (isolado CB 141).

Dentre aqueles testados, thiametoxam, imidaclopride e ciromazina não apresentaram efeito inibitório significativo no crescimento vegetativo e conidiogênese de $B$. bassiana, sendo as médias de tratamentos semelhantes à testemunha. Quando comparado ao presente trabalho, o tratamento espiromesifeno na dose média apresentou desenvolvimento vegetativo superior à testemunha, sendo que este fato pode estar relacionado à presença de substâncias que estimulariam a esporulação do fungo.

Alves et al. (1998) verificaram que alguns produtos fitossanitários químicos utilizados na cultura dos citros proporcionaram diminuição do diâmetro médio das colônias de Lecanicillium lecanii Zimm, B. bassiana e M. anisopliae, sem alteração no número de conídios produzidos, assemelhando-se aos resultados verificados no presente trabalho. 
Tabela 3. Classificação dos produtos fitossanitários químicos de acordo com a sua toxicidade.

\begin{tabular}{lccc}
\hline Ingrediente Ativo & Doses & IB $^{\mathbf{1}}$ & Classificaçã $^{\mathbf{1}}$ \\
\hline Piriproxifem (T) & 0,250 & 23,68 & Tóxico \\
Piriproxifem (T) & 0,275 & 22,07 & Tóxico \\
Piriproxifem (T) & 0,300 & 22,88 & Tóxico \\
Espiromesifeno & 0,400 & 48,66 & Moderadamente Tóxico \\
Espiromesifeno & 0,500 & 67,48 & Compatível \\
Espiromesifeno & 0,600 & 52,84 & Moderadamente Tóxico \\
Beta-cyfluthrin + Imidacloprido & 0,250 & 54,38 & Moderadamente Tóxico \\
Beta-cyfluthrin + Imidacloprido & 0,275 & 53,27 & Moderadamente Tóxico \\
Beta-cyfluthrin + Imidacloprido & 0,300 & 44,44 & Moderadamente Tóxico \\
Thiamethoxam + Lambdacialotrina & 0,250 & 37,07 & Tóxico \\
Imidacloprido & 0,250 & 40,84 & Tóxico \\
Piriproxifem (C) & 0,250 & 27,45 & Tóxico
\end{tabular}

${ }^{1}$ Rossi-Zalaf et al. (2008): 0 a 41 - Tóxico; 42 a 66 Moderadamente Tóxico; > 66 Compatível.

Tamai et al. (2002) avaliaram a toxicidade de 93 produtos fitossanitários para $B$. bassiana e, utilizando o teste $\mathrm{T}$ para determinação da compatibilidade (que não leva em consideração o efeito sobre a germinação), agruparam os resultados em quatro níveis de classificação: a) $22(23,6 \%)$ compatíveis, b) $5(5,4 \%)$ moderadamente tóxicos, c) $6(6,5 \%)$ tóxicos e d) 60 $(64,5 \%)$ muito tóxicos. Verificou-se que a distribuição dos produtos nos níveis de classificação foi bastante diferente entre as classes de produtos fitossanitários. Segundo os autores, o modo de ação do ingrediente ativo é possivelmente um dos principais fatores envolvidos no nível de toxicidade dos produtos fitossanitários aos fungos entomopatogênicos. Dentre os produtos testados por esses autores, piriproxifem (C) e piriproxifem (T) foram classificados como muito tóxicos, assemelhando-se aos resultados verificados na presente pesquisa, que os classificou como tóxicos.

Observa-se que a maioria dos produtos testados foi prejudicial ao fungo entomopatogênico B. bassiana. De acordo com Loureiro et al. (2002), ao se estabelecer uma estratégia de introdução conjunta desses fungos com os produtos fitossanitários químicos (controle associado), deve-se dar prioridade ao uso dos produtos que se mostraram menos prejudiciais e, portanto, mais compatíveis.

Os estudos in vitro têm a vantagem de expor ao máximo o microrganismo à ação do produto químico, fato que não ocorre em condições de campo, onde vários fatores servem de obstáculo a essa exposição. Assim, constatada a inocuidade de um produto em laboratório, espera-se que o mesmo seja compatível em campo. Por outro lado, a alta toxicidade de um produto in vitro nem sempre indica a sua elevada toxicidade em campo, mas sim a possibilidade da ocorrência de danos dessa natureza (Moino Junior e Alves, 1998).

Os resultados obtidos mostram que a ação dos produtos fitossanitários sobre o crescimento vegetativo e a produção de conídios variou em função da natureza química dos produtos. De acordo com Morris (1977), a presença de emulsificantes e outros aditivos contribuem com a incompatibilidade de produtos fitossanitários químicos aos entomopatógenos, representando um fator importante a ser controlado na elaboração de novas formulações comerciais de produtos.

\section{Conclusões}

Apenas o inseticida contendo piriproxifem na dose máxima não interferiu negativamente na produção de conídios.

Todos os inseticidas e doses testadas reduziram significativamente a germinação dos conídios.

Apenas o inseticida contendo espiromesifeno, na dose média, proporcionou crescimento vegetativo significativamente superior à testemunha, possibilitando sua classificação como compatível com o fungo $B$. bassiana.

\section{AGRADECIMENTOS}

À empresa Ballagro Agro Tecnologia, pela cedência do produto Ballvéria (Beauveria bassiana, isolado IBCB 66).

\section{Referências Bibliográficas}

Almeida, J. E. M.; Batista Filho, A.; Lamas, C.; Leite, L. G. 2003 Avaliação da compatibilidade de defensivos agrícolas na conservação de microrganismos entomopatogênicos no manejo do cafeeiro. Arquivos do Instituto Biológico, 70, 7984.

Alves, S. B. 1998. Controle Microbiano de Insetos. FEALQ.

Alves, S. B.; Moino Junior, A.; Almeida, J. E. M. 1998. Produtos Fitosanitários e Entomopatógenos, in: Alves, S.B., Controle Microbiano de Insetos. FEALQ, 217-238. 
Anderson, T. E.; Roberts, D. W. 1983. Compatibility of Beauveria bassiana isolates with insecticide formulations used in Colorado potato beetle (Coleoptera: Chrysomelidae) control. Journal of Economic Entomology, 76, 1437-1441.

Baldin, E. L. L.; Vendramin, J. D.; Lourenção A. L. 2005. Resistência de Genótipos de Tomateiro à mosca-branca Bemisia tabaci (Gennadius) Biótipo B (Hemiptera Aleyrodidae). Neotropical Entomology, 34, 435-441.

Faria, m. R, magalhães, b. P. 2001. Uso de fungos entomopatogênicos no Brasil. Biotecnologia, Ciência \& Desenvolvimento, 22, 18-21.

Lima, A. C. S.; Lara, F. M. 2004. Resistência de Genótipos de Soja à Mosca Branca Bemisia tabaci (Genn.) Biótipo B (Hemiptera: Aleyrodidae). Neotropical Entomology, 33, 7175 .

Loureiro, E. S., A. Moino Junior, Arnosti, A.; Souza G. C. 2002. Efeito de produtos fitossanitários químicos utilizados em alface crisântemo sobre fungos entomopatogênicos. Neotropical Entomology, 3, 263-269.

MAPA/AGROFIT. 2017. Sistemas de Agrotóxicos Fitossanitários $\quad-\quad$ Consulta $\quad$ Aberta. http://agrofit.agricultura.gov.br/agrofit/ (acesso em: 19. Mai 2017).

Moino Junior, A.; Alves, S. B. 1998. Efeito de Imidacloprid e Fipronil sobre Beauveria bassiana (Bals.) Vuill.e Metarhizium anisopliae (Metsch.) Sorok. e no comportamento de limpeza de Heterotermes tenuis (Hagen). Anais da Sociedade Entomológica do Brasil, 27, 611-619.

Morris, O. N. 1977. Compatibility of 27 chemical insecticides with Bacillus thuringiensis var. Kurstaki. The Canadian Entomologist, 109, 855-864.
Newsom, L. D.; Smith, S. F.; Whitcomb, W. H. 1976. Selective Pesticides and Selective Uses of Pesticides, in: Huffaker C. B.; Messenger, P. S., Theory and Practice of Biological Control. Academic Press, 565-591.

Onofre, S. B.; Miniuk, C. M.; Barros, N. M.; Azevedo, J. L. 2001. Growth and sporulation of Metarhizium flavoviride var. flavoviride on culture media and lighting regimes. Scientia Agricola, 58, 613-616.

Roberts, D. W.; Campbell, A. S. 1977. Stability of entomopathogenic fungi. Miscellaneous publications of the Entomological Society of America, 10, 19-75.

Rossi-Zalaf, L. S.; Alves, S. B.; Lopes, R. B.; Silveira Neto, S.; Tanzini, M. R. 2008. Interação de Micro-organismos Com Outros Agentes de Controle de Pragas e Doenças, in: ALVES, S.B; LOPES, R.B.(Eds.), Controle Microbiano de Pragas na América Latina: Avanços e Desafios. FEALQ, 279-302.

Silva, L. D.; Omoto C.; Bleicher, E.; Dourado, P. M. 2009. Monitoramento da suscetibilidade a inseticidas em populações de Bemisia tabaci (Gennadius) (Hemiptera: Aleyrodidae) no Brasil. Neotropical Entomology, 38, 116-125.

Sosa-Gomez, D. R. 2017. Seletividade de Agroquímicos Para Fungos Entomopatogênicos. http://www.cnpso.embrapa.br/download/artigos/seletiv_fung.p df (acesso em: 03 jun. 2017).

Tamai, M. R.; Alves S. B.; Lopes, R. B.; Faion, M.; Padulla, L. F. L. 2002. Toxicidade de Produtos Fitossanitários para Beauveria bassiana (Bals.) Vull. Arquivos do Instituto Biológico, 69, 89-96.

Yamamoto, P. T; Bassanezi, R. B. 2003. Seletividade de produtos fitossanitários aos inimigos naturais de pragas dos citrus. Revista Laranja, 24, 353-382. 